\title{
Subjective Evaluations of Fabric Wear Prickle Sensation by Using New Rating Scale
}

Rabie Ahmed Mohammed ASAD
\[ \mathrm{YU}^{1,3,2, \mathrm{c}} \]

${ }^{1}$ Key Laboratory of Textile Science \& Technology, Ministry of Education, Donghua

University, Shanghai, P. R. China 201620.

${ }^{2}$ Textile Materials and Technology Lab (TMT), Donghua University, Shanghai 201620, People's Republic of China.

${ }^{3}$ Faculty of Textiles, University of Gezira, Wad-Medani, P.o. Box 20, Sudan.

${ }^{4}$ Department of Manufacturing, Industrial and Textile Engineering, School of

Engineering, Moi University, P.o. Box 3900-30100, Eldoret, Kenya.

arabieasad@yahoo.com, ㅁnkip2007@yahoo.com, wdyu@dhu.edu.cn (Corresponding author)

Keywords: Fabric, Prickle, human skin, body sensation, rating scale

\begin{abstract}
In this study, prickle sensation was investigated for wear fabrics having different compositions and physical surface characteristics using 1-7 rating scale. Rating score test for prickle sensory discomfort wear fabric test was conducted on 31 subjects. It was found that prickleness sensation arise during skin-fabric contact are mostly related to the fiber and surface roughness characteristics of fabrics. It was also found that there are highly significant correlation coefficients between the evaluation results of using the different body parts.
\end{abstract}

\section{Introduction}

The assessment of handle characteristics is an important tool not only for innovative product development, but also to investigate, define and safeguard the original quality of traditional fabrics [1]. Fabric-evoked prickle has been identified as one of the most irritating discomfort sensations for garments worn next-to-skin. Prickleness is experienced when the fabric is patted or pressed onto human skin, but it is not felt on a hairless skin such as on palms and fingers, since pain nerve endings are very close to the surface in hairy skin but not in glabrous skin [2,3]. Prickle, a specific and sometimes irritating component of the tactile aspect of comfort, has been the focus of a number of studies $[2,4,5]$. All of these studies carried out on different fabrics having different degrees of prickleness data under different levels of studies have common results that, perception of prickle depends on fiber properties especially fiber diameter and length. In this study, different human body parts of 31 healthy males and females were investigate the fabric-evoked prickle sensations created by eight woven fabrics differing in their materials and physical/constructional properties.

\section{Materials and methods}

Fabrics samples. Eight fabrics, most of them made from natural fibers, have been chosen in this study for their similarities in production methods and final use. Fabric samples namely cotton, cashmere, linen, hemp, ramie, jute, wool, and cotton/polyester. Before the subjective test started, all samples were conditioned for a minimum of $48 \mathrm{hr}$ under standard atmospheric conditions $(20 \pm 2){ }^{0} \mathrm{C}$ temperature, $(65 \pm 2) \%$ relative humidity.

Subjects. Thirty one undergraduate and postgraduate students from eleven countries at Donghua University were selected to participate in subjective sensory evaluation of prickleness of textiles wear fabrics. The subjects were selected among them are professional sportsmen having similar physiological characteristics (ages between 19 and 38 years, heights between 155 and $194 \mathrm{~cm}$, and weights between 48 and $110 \mathrm{~kg}$ ). 
Human body parts. It is well known in physiology that the human body and several of its members also vary in feeling from the resulting sense when it is connected to various materials. So in this study, the prickle sensations of the above eight fabrics was evaluated by using six different human body parts namely right forearms, back neck, upper back, abdomen, right side waist, and inner right thigh of the subject.

Subjective rating scales used for fabric-evoked prickle sensations scores. A total of seven sensations were rated at defined periods during each test session, as listed in Table 2. The 1-7 rating scale is shown in Fig. 1 were used to gather the prickly sensations of human body. Each combination of a test part and a rating scale is described in Table 2, and six tests were carried out separately.

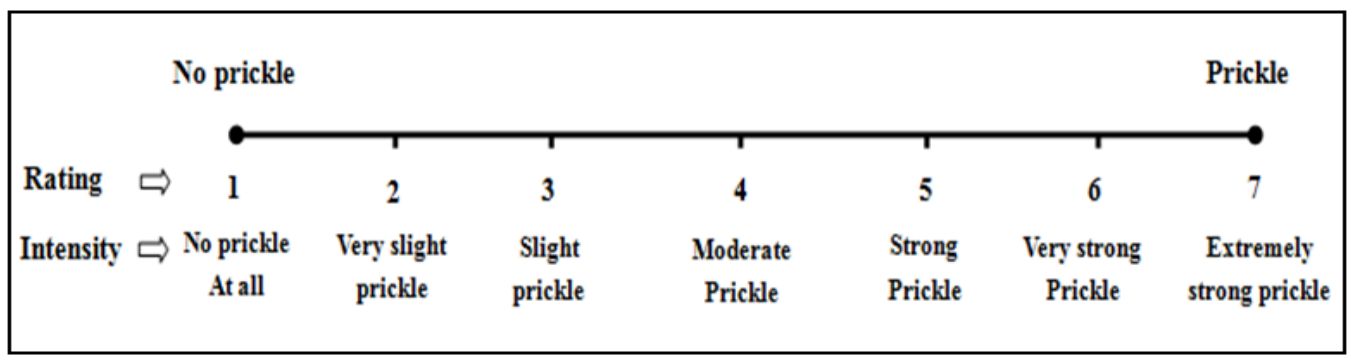

Fig. 1 The 1-7 rating scales used for fabric-evoked prickle sensations

Test protocol of Fabric-evoked prickle sensory test.The tests protocol was conducted for a period of nearly to twenty weeks. The prickleness test was divided into 16 trials. In order to get good results and to avoid the errors that result from the subject psychological state, the test protocol consisted as follow:

The right hand moved the fabric sample with constant pressure back and forth and also from right to left for 15 seconds to detect the prickle sensory. Prickle sensation grade measurement was started after the fabric was placed on the different subject body parts one by one. The prickle degree caused from the skin-fabric contact was gathered by the continuous measurements. The records made after the subjects were asked to fill a questionnaire by making comparative evaluation about prickliness comfort sensation of the subject body stimuli.

Table 2 Rating scale used to describe each of the sensations in each period of prickle sensation

\begin{tabular}{cll}
$\begin{array}{c}\text { Prickle } \\
\text { rating scale }\end{array}$ & $\begin{array}{c}\text { Tactile prickle } \\
\text { attributes }\end{array}$ & \multicolumn{1}{c}{ Human stimuli when in contact to the fabric } \\
\hline $\mathbf{1}$ & No prickle at all & $\begin{array}{l}\text { The fabric surface is soft, smooth and comfortable when in } \\
\text { contact human skin. }\end{array}$ \\
\hline $\mathbf{3}$ & Slight prickle & $\begin{array}{l}\text { The fabric surface has a lot of protruding fibers which irritates } \\
\text { the skin, but can be tolerated when in contact human skin. }\end{array}$ \\
\hline $\mathbf{5}$ & $\begin{array}{l}\text { Moderate } \\
\text { Prickle }\end{array}$ & $\begin{array}{l}\text { At this intensity, the fabric surface has normal freckles itch } \\
\text { and pain impact on human skin when it contacts the fabric. } \\
\text { But can be tolerated when in contact. }\end{array}$ \\
\hline $\mathbf{6}$ & Strong prickle & $\begin{array}{l}\text { The fabric evokes a desire to scratch and the surface has a lot } \\
\text { of protruding fibers which causes irritation and slight itching } \\
\text { of the human skin, but can be tolerated when in contact. }\end{array}$ \\
\hline $\mathbf{7}$ & $\begin{array}{l}\text { Very } \\
\text { Prickle }\end{array}$ & $\begin{array}{l}\text { The fabric is harsh or abrasive on the skin, its surface is very } \\
\text { rough and arouses strong stimulation to the skin, in addition, it } \\
\text { makes the skin itchy, irritating and causes scratchiness when } \\
\text { in contact with the human skin. }\end{array}$ \\
\hline Extremely & strong & $\begin{array}{l}\text { The fabric evokes a sensation of discomfort and the fabric } \\
\text { surface is extremely rough, stiff and scratching the skin a lot, } \\
\text { which is intolerable. The fabric immediately evokes sensation } \\
\text { and makes very gentle prickle pain. }\end{array}$ \\
\hline
\end{tabular}




\section{Result and discussion}

Wear fabrics prickle score for different body parts. Relative six body parts fabric-evoked prickle sensory responses were obtained as described above and the results were plotted in Fig. 2 . For each subject, the average of the scores for the three samples of each fabric was used as the total prickle score given by the difference subjects. Therefore, for each fabrics the average of the prickle scores given by the 31 subjects was used to calculate the mean, STD, and variance of prickliness score of the all wear fabrics samples.

The results of all trials using trained and experienced judges confirm that the different worn fabrics were perceived as having different prickle score. This is consistent with the different hypothesis that prickle is related to the presence and quantity of coarse fiber ends pressing against the skin. It is interesting that the subjects were able to distinguish the degree of prickle sensations of the fabrics manufactured from the different fiber samples, that is, real differences in prickle score are detectable. Likewise, the panel of trained subjects could not distinguish the relative prickle score of the cotton, cashmere, and cotton/polyester fabrics and these fabrics were observed to have a significantly lower level of prickle score than the other fabrics especially wool, ramie and jute wear fabric.

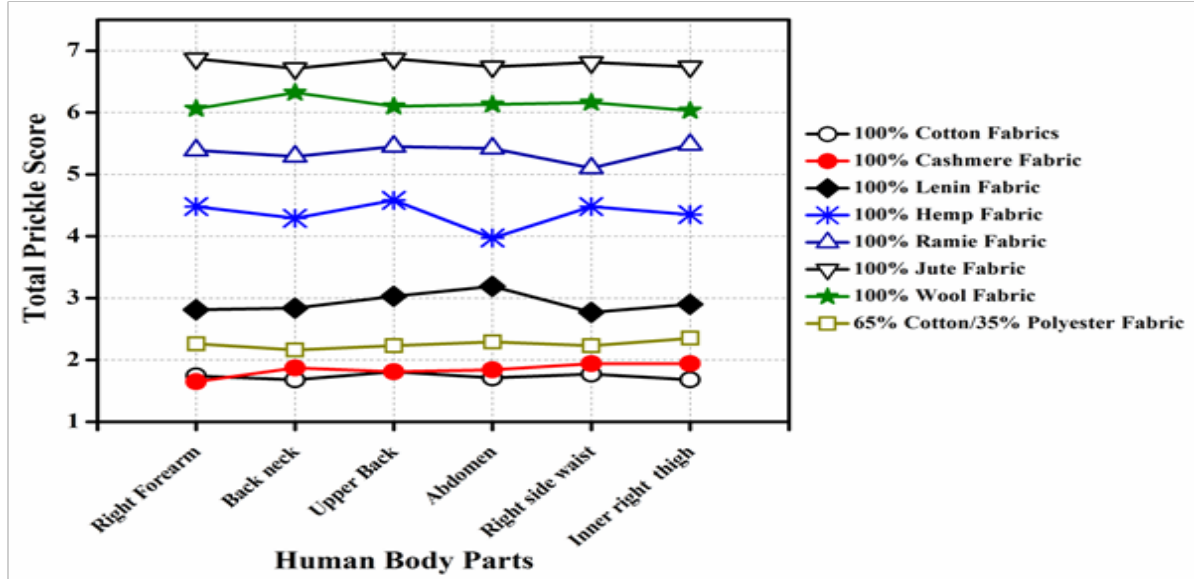

Fig. 2 Comparison of prickle score rating between fabric types on different human body point

Statistical analysis of total prickleness score. Correlation analysis was carried out to test the consistency level of the prickle score evaluation results obtained from the different human body parts. Here, we regarded the prickliness score of each fabric tested on one part and using one rating scale as an individual observation. Therefore, ANOVA analysis was carried out to investigate if there were statistical differences on the CV values occurred in different part tests for prickle sensory and for all fabrics samples. From the results, it can be seen that the value of $\boldsymbol{F}$ is lower than $\boldsymbol{F}_{\mathbf{0 . 0 5}}$ in the observations of prickliness score for all fabric samples. This result indicates that there is no statistical difference among the CV values occurred in different body parts tests for prickle. There is enough evidence to support the claim that there is no difference among the means.

\section{Conclusion}

In this study the mean scores for all fabrics indicated that the cotton, cashmere, cotton/polyester fabric was significantly less prickly sensation than the ramie, wool, and jute fabric and that cotton, cashmere, and cotton/polyester fabrics were less prickly detection and it was more comfortable fabric. Therefore, the different human body parts prickle test with 1-7 rating scale was recommended in evaluating fabric-evoked prickle for its convenience and sensitivity.

\section{References}

[1] L. Yi, The science of clothing comfort. Textile Progress, 31(2001) 1-135.

[2] G. Song: Improving comfort in clothing, Cambridge, UK: Woodhead Publishing. 1(2011)23-78. 
[3] G. R. S. Naylor, J.H. Stanton and J. Speijers: Skin comfort of base layer wool garments. Part 2: fiber diameter effects on fabric and garment prickle. Textile Research J., 2014: p. 0040517514523174.

[4] R. K. Garnsworthy, R. L. Gully, R. P. Kandiah, P. Kenins, R. J. Mayfield and R. A. Westerman: Understanding the causes of prickle and itch from the skin contact of fabrics. CSIRO Division of Wool Technology Report, . 64 (1988).

[5] R. K. Garnsworthy, R. L. Gully, P. Kenins and R. A. Westerman:., Transcutaneous electrical stimulation and the sensation of prickle. Journal of neurophysiology, 59(1988) 1116-1127. 